\title{
$\mathbb{A}$ Economics Bulletin
}

\section{Volume 30, Issue 1}

\section{Money and price dynamics in a market with strategic bargaining}

\author{
Noritaka Kudoh \\ Hokkaido University
}

\begin{abstract}
This paper studies a strategic bargaining model of money and prices to complement the results reported in Coles and Wright (1998). The probability of a bargaining breakdown is chosen to be consistent with market conditions in the spirit of Rubinstein and Wolinsky (1985). The unique monetary steady state coincides with the one under asymmetric Nash bargaining. The dynamics of the price level are determined without any reference to the value of search. The dynamic properties of the model resemble those of traditional monetary models.
\end{abstract}

I would like to thank Akihiko Matsui and Naohito Abe for their helpful comments.

Citation: Noritaka Kudoh, (2010) "Money and price dynamics in a market with strategic bargaining", Economics Bulletin, Vol. 30 no.1 pp. 709-719.

Submitted: Jan 12 2010. Published: March 10, 2010. 


\section{Introduction}

The purpose of this paper is to study the dynamics of the price level using a model where traders within the model choose the prices of their goods rather than relying on the Walrasian auctioneer. To that end, this paper provides results that complement those reported in Coles and Wright (1998). In particular, it introduces a random proper version of Rubinstein and Wolinsky's (1985) bargaining game into Coles and Wright's (1998) monetary search model.

In Coles and Wright (1998), the price dynamics are derived using a random-proposer version of Rubinstein's (1982) bargaining game, in which there is no bargaining breakdown. They also considered a bargaining process similar to Rubinstein and Wolinsky (1985). However, their choice of the probability of a bargaining breakdown was arbitrary. In addition, they focused on the price dynamics with Rubinstein's (1982) bargaining game.

In this paper, I modify the model of Coles and Wright (1998) so that the probability of a bargaining breakdown is chosen to be consistent with market conditions (i.e., the numbers of buyers and sellers in the market). In other words, the bargaining process reflects additional information about the market.

The steady state bargaining outcome coincides with the one under asymmetric Nash bargaining. There is a unique monetary steady state characterized as a "source" and a hyperinflationary path leading to the non-monetary steady state, just as in the traditional monetary models, in which money is valued because it is in the utility function (Obstfeld and Rogoff, 1983).

\section{The Model}

The economy is populated by a continuum of infinitely lived agents whose measure is normalized to unity. There are $k>2$ types of goods and $k$ types of agents. Type $i$ agent is defined as an agent whose consumption good is indexed by $i$ and production good is $i+1$ (modulo $k$ ). The instantaneous utility from consuming $q$ units of the good is $U(q)$, where $U(0)=0, U^{\prime}>0$, and $U^{\prime \prime} \leq 0$. If agent $i$ is to produce, then the utility cost of producing $q$ units of the good is $c^{i}(q)=c(q)$ for any $i$, with $c(0) \geq 0, c^{\prime}>0$, and $c^{\prime \prime} \geq 0$. A fraction $M$ of the total population has one unit of fiat money (i.e., buyers). The complementary fraction $1-M$ of the population is endowed with a production opportunity (i.e., sellers). No agent is allowed to hold more than one unit of money. Since one unit of fiat money is exchanged for $q$ units of a certain commodity, $1 / q \equiv p$ is the price level.

Time is continuous, but for convenience a sufficiently short length of time $\Delta$ is considered as a period. The Poisson arrival rate at which an agent meets another is $\alpha$ so that during a small period of time $\Delta$ the probability that a buyer meets a seller is $\alpha \Delta(1-M)$. Upon meeting, a match is formed if and only if the seller has a good that is acceptable to the buyer. Similarly, the probability that a seller meets a buyer in a short period of length $\Delta$ is $\alpha \Delta M$.

With probability $\alpha \Delta(1-M) / k$ the buyer meets a seller who has his/her consumption good. In this case, the buyer consumes the good and becomes a seller. With probability 
$1-\alpha \Delta(1-M) / k$ the buyer finds no match and continues to search. Let $V_{b}(t)$ denote the value of search as a buyer and $V_{s}(t)$ denote the value of search as a seller. The rate of time preference is $r>0$.

Throughout the paper, I impose $\alpha / k=1$ for brevity. Then, the buyer's value of search is given recursively as $V_{b}(t)=(1+r \Delta)^{-1}\left\{(1-M) \Delta\left[E U(q(t+\Delta))+V_{s}(t+\Delta)\right]+(1-\Delta(1-\right.$ $\left.M)) V_{b}(t+\Delta)+o(\Delta)\right\}$, where $E U(q(t+\Delta))$ is the expected utility from consumption and $o(\Delta) / \Delta \rightarrow 0$ as $\Delta \rightarrow 0$. For rationality, it is necessary that all trades are voluntary. This requires that $E U(q(t+\Delta))+V_{s}(t+\Delta) \geq V_{b}(t+\Delta)$. Similarly, the seller's value of search is given by: $V_{s}(t)=(1+r \Delta)^{-1}\left\{\Delta M\left[-E c(q(t+\Delta))+V_{b}(t+\Delta)\right]+(1-\Delta M) V_{s}(t+\Delta)+o(\Delta)\right\}$, where $E c(q(t+\Delta))$ is the expected cost of production. Rationality requires $-E c(q(t+\Delta))+$ $V_{b}(t+\Delta) \geq V_{s}(t+\Delta)$.

Let $\Delta \rightarrow 0$ to obtain the following:

$$
\begin{aligned}
& r V_{b}=(1-M)\left(E U(q)+V_{s}-V_{b}\right)+\dot{V}_{b}, \\
& r V_{s}=M\left(-E c(q)+V_{b}-V_{s}\right)+\dot{V}_{s},
\end{aligned}
$$

where $\dot{V}_{b} \equiv \partial V_{b} / \partial t$ and $\dot{V}_{s} \equiv \partial V_{s} / \partial t$.

The bargaining game employed in this paper is a random proposer version of Rubinstein and Wolinsky (1985). In any period in which a buyer and a seller are matched, one of them is selected randomly to make an offer: the buyer makes an offer with probability $\pi_{b}$, and the seller makes an offer with probability $\pi_{s}=1-\pi_{b}$. After an offer is made, the respondent may either accept it or search for a new bargaining partner. Following Rubinstein and Wolinsky (1985), the present model adopts a bargaining game with breakdowns. Thus, if a new bargaining partner arrives during the bargaining process, then the current bargaining partner will be discarded in favor of the new one.

I seek a bargaining equilibrium similar to the immediate trade equilibrium (ITE) considered by Coles and Wright (1998) in which there exist reservation values $q_{s}(t)$ and $q_{b}(t)$ such that all sellers accept any offer $q \leq q_{s}(t)$ and all buyers accept any offer $q \geq q_{b}(t)$. In addition, I follow Rubinstein and Wolinsky (1985) to assume that the strategies of the agents are semi-stationary, in which a trader submits the same offer or reply, irrespective of who his/her partner will be. See also Osborne and Rubinstein (1990, p.141.).

If a seller is to make an offer, he/she chooses $q_{b}(t)$ such that a buyer is indifferent between accepting and rejecting the offer, given the value functions $V_{b}$ and $V_{s}$ and the length of the bargaining period $\Delta$. The seller must take into account the following. The buyer meets a new seller with probability $\alpha \Delta(1-M)$. Given that a match occurs, with probability $1 / k$ this new agent turns out to be a seller who has the buyer's consumer good. In this case, the buyer will discard his/her current bargaining partner in favor of his/her new partner and receive the expected payoff $E U(q(t+\Delta))+V_{s}(t+\Delta)$, where the expected utility from consumption satisfies $E U(q(t+\Delta))=\pi_{s} U\left(q_{b}(t+\Delta)\right)+\pi_{b} U\left(q_{s}(t+\Delta)\right)$ because in the next round the seller will make an offer $q_{b}(t+\Delta)$ with probability $\pi_{b}$ and the buyer will make an offer $q_{s}(t+\Delta)$ with probability $\pi_{s}$. With probability $(1-\alpha \Delta(1-M) / k)(1-\alpha \Delta M / k)$ neither the buyer nor the seller will find a new partner and they will continue to be matched in the next bargaining stage. In the next stage, the buyer makes a new offer with probability $\pi_{b}$, and the 
seller makes a new offer with probability $\pi_{s}$. With probability $(1-\alpha \Delta(1-M) / k) \alpha \Delta M / k$ the buyer will not find a new bargaining partner and the seller will find one in the interval $(t, t+\Delta)$. In that case, the seller will walk away and the buyer will be left with the value of being unattached, $V_{b}(t+\Delta)$.

Thus, with $\alpha / k=1$, the seller's offer $\left\{q_{b}(t)\right\}_{t=0}^{\infty}$ must satisfy:

$$
\begin{aligned}
U\left(q_{b}(t)\right)+V_{s}(t)= & \frac{1}{1+r \Delta}\left\{\Delta(1-M)\left[E U(q(t+\Delta))+V_{s}(t+\Delta)\right]\right. \\
& +(1-\Delta(1-M))(1-\Delta M)\left[E U(q(t+\Delta))+V_{s}(t+\Delta)\right] \\
& \left.+(1-\Delta(1-M)) \Delta M V_{b}(t+\Delta)+o(\Delta)\right\},
\end{aligned}
$$

where the right-hand side of (3) is the expected value of rejecting $q_{b}(t)$.

Similarly, when a buyer is to make an offer, he/she chooses his/her offer such that a seller is indifferent between accepting $q_{s}(t)$ and rejecting it. Thus, the buyer's offer $\left\{q_{s}(t)\right\}_{t=0}^{\infty}$ must satisfy:

$$
\begin{aligned}
-c\left(q_{s}(t)\right)+V_{b}(t)= & \frac{1}{1+r \Delta}\left\{\Delta M\left[-E c(q(t+\Delta))+V_{b}(t+\Delta)\right]\right. \\
& +(1-\Delta(1-M))(1-\Delta M)\left[-E c(q(t+\Delta))+V_{b}(t+\Delta)\right] \\
& \left.+\Delta(1-M)(1-\Delta M) V_{s}(t+\Delta)+o(\Delta)\right\} .
\end{aligned}
$$

Note that, as is clear from $(3)$ and $(4), q_{b}(t)$ and $q_{s}(t)$ are not only functions of $t$, but also functions of $\Delta$. In what follows, I focus on the limit as $\Delta \rightarrow 0$, and derive a single differential equation that describes the price level.

Proposition 1 In the limit as $\Delta \rightarrow 0, \lim _{\Delta \rightarrow 0} q_{b}(t)=\lim _{\Delta \rightarrow 0} q_{s}(t) \equiv q(t)$ for any $t$, and the bargaining outcome satisfies

$$
\frac{\pi_{b} U^{\prime}(q)}{\pi_{s} c^{\prime}(q)}=\frac{(M+r) U(q)-M V_{b}+(M+r) V_{s}-U^{\prime}(q) \dot{q}-\dot{V}_{s}}{-(1-M+r) c(q)-(1-M) V_{s}+(1-M+r) V_{b}+c^{\prime}(q) \dot{q}-\dot{V}_{b}} .
$$

In steady state, it satisfies

$$
\frac{U^{\prime}(q)}{c^{\prime}(q)}=\frac{\pi_{s}}{\pi_{b}} \frac{U(q)+V_{s}-V_{b}}{-c(q)+V_{b}-V_{s}}
$$

Proof. See the Appendix.

Consider the following asymmetric Nash bargaining problem: $\max _{q}\left[-c(q)+V_{b}-T_{s}\right]^{\theta}[U(q)+$ $\left.V_{s}-T_{b}\right]^{1-\theta}$, from which the bargaining outcome satisfies

$$
\frac{U^{\prime}(q)}{c^{\prime}(q)}=\frac{\theta}{1-\theta} \frac{U(q)+V_{s}-T_{b}}{-c(q)+V_{b}-T_{s}},
$$

where $T_{b}$ and $T_{s}$ are the threats for the buyer and the seller, respectively, and $\theta$ represents the exogenous bargaining power of the seller. The threat represents the payoff when the negotiation fails. Coles and Wright (1998) assumed $\left(T_{b}, T_{s}\right)=(0,0)$, which corresponds to 
Rubinstein (1982). This implies that the bargaining parties negotiate over time as if there was nobody else in the market, and this specification is somewhat ad hoc.

The bargaining outcome (6) corresponds to the Nash solution with $\left(T_{b}, T_{s}\right)=\left(V_{b}, V_{s}\right)$ and $\theta=\pi_{s}$. In other words, in the steady state, the economy corresponds to the one under asymmetric Nash bargaining where the exogenous bargaining power is given by the probability of making an offer, and the threat equals the value of search.

\section{Unique Monetary Equilibrium}

From (1) and (2), we have:

$$
\dot{V}=(1+r) V-(1-M) U(q)-M c(q),
$$

where $V \equiv V_{b}-V_{s}$. Use (1) and (2) to eliminate $\dot{V}_{b}$ and $\dot{V}_{s}$ from (5) to obtain:

$$
\dot{q}=\pi_{s} \frac{(M+r) U(q)-M c(q)}{U^{\prime}(q)}-\pi_{b} \frac{(1-M) U(q)-(1-M+r) c(q)}{c^{\prime}(q)} \equiv G(q) .
$$

A monetary equilibrium is given by the sequence of $V$ and $q$ that satisfy (8), (9), and the rationality condition, $c(q) \leq V \leq U(q)$ or $(1+r) c(q) \leq(1-M) U(q)+M c(q) \leq(1+r) U(q)$. The key difference from Coles and Wright (1998) is that (9) is independent of $V$. In other words, when the probability of a bargaining breakdown is chosen to be consistent with the market condition, the path of the price level is determined without any reference to the value of search. This result has an important implication. Consider Figure 1, in which the steady state monetary equilibrium is given by the intersection between the $\dot{q}=0$ locus and the $\dot{V}=0$ locus. As shown in the figure, the monetary steady state is unique.

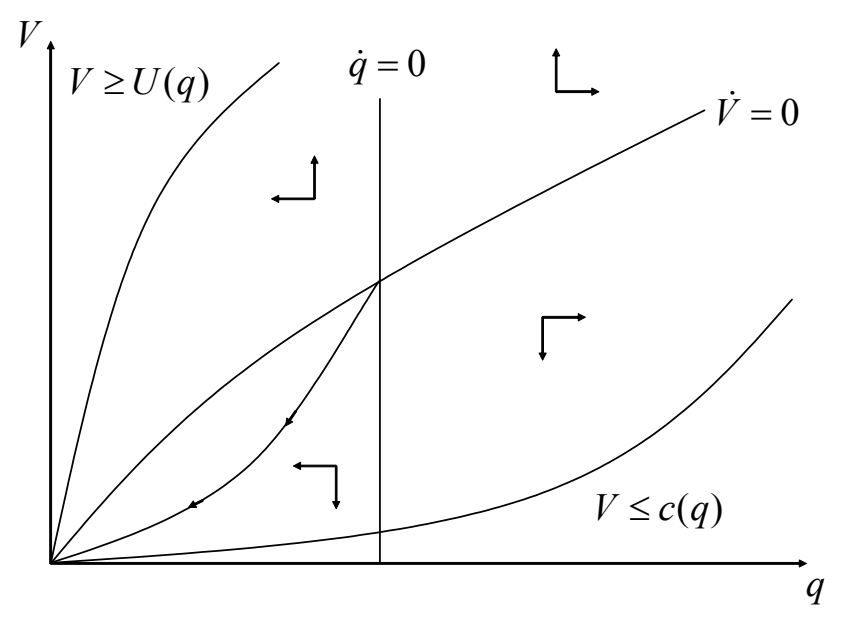

Figure 1

Having established uniqueness, I turn to the issue of stability.

Proposition 2 The monetary steady state is a source. 
Proof. In the Appendix.

Interestingly, the unique monetary steady state is characterized by a "source" (see, for example, Azariadis, 1993). This implies that, as in the traditional models of money (Brock, 1975; Obstfeld and Rogoff, 1983) there is a path leading to the non-monetary equilibrium $(0,0)$. Absence of a state variable suggests that there is a continuum of self-fulfilling "hyperinflationary" non-stationary equilibria, just as in the traditional models. In addition, since all other divergent paths will eventually violate one of the rationality conditions, there is a unique monetary equilibrium, in which the economy stays in the monetary steady state at any moment in time.

To highlight the (rather stark) difference between this economy and the one presented in Coles and Wright (1998), let us introduce a fixed cost of production, $c(0)=\bar{c}>0$, just as in Coles and Wright (1998), who showed that with this fixed cost there are two monetary steady states, and studied nonlinear dynamics around the steady states. Multiple equilibria are possible in their model because their $\dot{q}=0$ locus is an upward-sloping curve. As shown in Figure 1, the $\dot{q}=0$ locus is a vertical line in this model. This implies that the number of monetary equilibria is invariant to the introduction of a fixed cost.

What does a fixed cost do in this economy? Since any steady state must satisfy $(1+r) V=$ $(1-M) U(q)+M c(q)$, it is easy to verify that as $q \rightarrow 0, V \rightarrow(1+r)^{-1}[(1-M) U(0)+M c(0)]=$ $(1+r)^{-1} M \bar{c}<\bar{c}=c(0)$, which violates the seller's rationality $V \geq c(q)$. Thus, the nonmonetary steady state $(q=0)$ cannot be an equilibrium. In other words, with a fixed cost, the monetary steady state is the only equilibrium. Interestingly, in this economy, a fixed cost simply rules out the non-monetary equilibrium without introducing multiple monetary equilibria. To be more specific, the hyperinflationary paths are ruled out by a fixed cost. This resembles the way in which hyperinflationary paths are ruled out in the traditional monetary models (Brock, 1975; Obstfeld and Rogoff, 1983).

\section{Conclusion}

An interesting implication of the model in this paper is that even in a model where money is valued because of search frictions, the path of the price level is fully determined without any reference to the value of search. This suggests an interesting dichotomy between the unit-ofaccount role of money and the medium-of-exchange role of money. This property is new in the literature. Important future research is to explore the degree to which this dichotomy is true. This line of inquiry will contribute to the recent debate on whether the price dynamics can be understood without explicitly modeling monetary frictions (Woodford, 2003). 


\section{Appendix}

\section{A Proof of Proposition 1}

The proof requires several steps.

Lemma $3 V_{b}$ and $V_{s}$ are uniformly bounded.

Proof. Let $\widehat{q}$ be the solution to $U(\widehat{q})=c(\widehat{q})$. From rationality $c(q) \leq V \leq U(q)$ it is evident that $q(t+\Delta) \leq \widehat{q}$ holds for all $t$ and $\Delta$. In other words, $q$ is bounded by $\widehat{q}$. Thus, the maximum possible lifetime utility an agent can get is $U(\widehat{q}) / r$. Thus, $V_{b}$ and $V_{s}$ are bounded from above. Furthermore, the value functions are bounded below by 0 . Therefore, $V_{b}$ and $V_{s}$ are uniformly bounded.

Lemma $4 \varepsilon(t) \equiv q_{s}(t)-q_{b}(t)$ converges to zero at rate $\Delta$ as $\Delta \rightarrow 0$.

Proof. Multiply (3) by $(1+r \Delta)$, rearrange terms, divide both sides by $\Delta$, and take the limit as $\Delta \rightarrow 0$ to obtain

$$
\begin{aligned}
& \lim _{\Delta \rightarrow 0} \frac{U\left(q_{b}(t)\right)-\left[\pi_{s} U\left(q_{b}(t+\Delta)\right)+\pi_{b} U\left(q_{s}(t+\Delta)\right)\right]}{\Delta} \\
= & -M\left[E U(q(t))+V_{s}(t)\right]-r\left[U\left(q_{b}(t)\right)+V_{s}(t)\right]+M V_{b}(t)+\dot{V}_{s} .
\end{aligned}
$$

Similarly, multiply (4) by $(1+r \Delta)$, rearrange terms, divide both sides by $\Delta$, and take the limit as $\Delta \rightarrow 0$ to obtain

$$
\begin{aligned}
& \lim _{\Delta \rightarrow 0} \frac{-c\left(q_{s}(t)\right)+\pi_{s} c\left(q_{b}(t+\Delta)\right)+\pi_{b} c\left(q_{s}(t+\Delta)\right)}{\Delta} \\
= & -(1-M)\left[-E c(q(t))+V_{b}(t)\right]-r\left[-c\left(q_{s}(t)\right)+V_{b}(t)\right]+(1-M) V_{s}(t)+\dot{V}_{b} .
\end{aligned}
$$

Lemma 3 implies that (3) and (4) are finite for any $\Delta$. Thus, the left-hand sides of (10) and (11) are bounded. Consider the left-hand side of (10). Since $U\left(q_{b}(t)\right)-\pi_{s} U\left(q_{b}(t+\Delta)\right)-$ $\pi_{b} U\left(q_{s}(t+\Delta)\right)=\pi_{b}\left[U\left(q_{b}(t)\right)-U\left(q_{s}(t)\right)\right]-\pi_{s}\left[U\left(q_{b}(t+\Delta)\right)-U\left(q_{b}(t)\right)\right]-\pi_{b}\left[U\left(q_{s}(t+\Delta)\right)-\right.$ $\left.U\left(q_{s}(t)\right)\right]$, it is easy to show that

$$
\begin{aligned}
& \lim _{\Delta \rightarrow 0} \frac{U\left(q_{b}(t)\right)-\left[\pi_{s} U\left(q_{b}(t+\Delta)\right)+\pi_{b} U\left(q_{s}(t+\Delta)\right)\right]}{\Delta} \\
= & \lim _{\Delta \rightarrow 0}\left[\begin{array}{c}
\pi_{b} \frac{U\left(q_{b}(t)\right)-U\left(q_{s}(t)\right)}{\Delta}-\pi_{s} \frac{U\left(q_{b}(t+\Delta)\right)-U\left(q_{b}(t)\right)}{q_{b}(t+\Delta)-q_{b}(t)} \frac{q_{b}(t+\Delta)-q_{b}(t)}{\Delta} \\
-\pi_{b} \frac{U\left(q_{s}(t+\Delta)\right)-U\left(q_{s}(t)\right)}{q_{s}(t+\Delta)-q_{s}(t)} \frac{q_{s}(t+\Delta)-q_{s}(t)}{\Delta}
\end{array}\right] \\
= & \pi_{b} \lim _{\Delta \rightarrow 0} \frac{U\left(q_{b}\right)-U\left(q_{s}\right)}{\Delta}-\pi_{s} U^{\prime}\left(q_{b}\right) \dot{q}_{b}-\pi_{b} U^{\prime}\left(q_{s}\right) \dot{q}_{s} .
\end{aligned}
$$


Similarly, the left-hand side of (11) can be rewritten as

$$
\begin{aligned}
& \lim _{\Delta \rightarrow 0} \frac{-c\left(q_{s}(t)\right)+\left[\pi_{s} c\left(q_{b}(t+\Delta)\right)+\pi_{b} c\left(q_{s}(t+\Delta)\right)\right]}{\Delta} \\
= & \lim _{\Delta \rightarrow 0}\left[\begin{array}{c}
-\pi_{s} \frac{c\left(q_{s}(t)\right)-c\left(q_{b}(t)\right)}{\Delta}+\pi_{s} \frac{c\left(q_{b}(t+\Delta)\right)-c\left(q_{b}(t)\right)}{q_{b}(t+\Delta)-q_{b}(t)} \frac{q_{b}(t+\Delta)-q_{b}(t)}{\Delta} \\
+\pi_{b} \frac{c\left(q_{s}(t+\Delta)\right)-c\left(q_{s}(t)\right)}{q_{s}(t+\Delta)-q_{s}(t)} \frac{q_{s}(t+\Delta)-q_{s}(t)}{\Delta}
\end{array}\right] \\
= & -\pi_{s} \lim _{\Delta \rightarrow 0} \frac{c\left(q_{s}\right)-c\left(q_{b}\right)}{\Delta}+\pi_{s} c^{\prime}\left(q_{b}\right) \dot{q}_{b}+\pi_{b} c^{\prime}\left(q_{s}\right) \dot{q}_{s} .
\end{aligned}
$$

Use (12) and (13) to rewrite (10) and (11) as

$$
\begin{aligned}
\pi_{b} \lim _{\Delta \rightarrow 0} \frac{U\left(q_{b}\right)-U\left(q_{s}\right)}{\Delta}= & -M\left[E U(q)+V_{s}\right]-r\left[U\left(q_{b}\right)+V_{s}\right]+M V_{b} \\
& +\pi_{s} U^{\prime}\left(q_{b}\right) \dot{q}_{b}+\pi_{b} U^{\prime}\left(q_{s}\right) \dot{q}_{s}+\dot{V}_{s}, \\
\pi_{s} \lim _{\Delta \rightarrow 0} \frac{c\left(q_{b}\right)-c\left(q_{s}\right)}{\Delta}= & -(1-M)\left[-E c(q)+V_{b}\right]-r\left[-c\left(q_{s}\right)+V_{b}\right] \\
& +(1-M) V_{s}-\pi_{s} c^{\prime}\left(q_{b}\right) \dot{q}_{b}-\pi_{b} c^{\prime}\left(q_{s}\right) \dot{q}_{s}+\dot{V}_{b},
\end{aligned}
$$

Lemma 3 implies that the right-hand sides above are bounded. In other words, $\lim _{\Delta \rightarrow 0}\left[U\left(q_{b}\right)-\right.$ $\left.U\left(q_{s}\right)\right] / \Delta$ and $\lim _{\Delta \rightarrow 0}\left[c\left(q_{b}\right)-c\left(q_{s}\right)\right] / \Delta$ have limits, and denote them as $\Lambda_{b}$ and $\Lambda_{s}$, respectively. This also implies that $\varepsilon(\Delta) \rightarrow 0$ as $\Delta \rightarrow 0$. Furthermore, since $U(\cdot)$ and $c(\cdot)$ are differentiable,

$$
\lim _{\varepsilon(\Delta) \rightarrow 0} \frac{U\left(q_{s}(t)+\varepsilon(\Delta)\right)-U\left(q_{s}(t)\right)}{\varepsilon(\Delta)} \text { and } \lim _{\varepsilon(\Delta) \rightarrow 0} \frac{c\left(q_{s}(t)+\varepsilon(\Delta)\right)-c\left(q_{s}(t)\right)}{\varepsilon(\Delta)}
$$

have limits, and denote them by $U^{\prime}\left(q_{s}\right)$ and $c^{\prime}\left(q_{s}\right)$, respectively. Thus,

$$
\begin{aligned}
& \Lambda_{b}=\pi_{b} \lim _{\Delta \rightarrow 0} \frac{U\left(q_{s}(t)+\varepsilon(\Delta)\right)-U\left(q_{s}(t)\right)}{\varepsilon(\Delta)} \frac{\varepsilon(\Delta)}{\Delta}=\pi_{b} U^{\prime}\left(q_{s}\right) \lim _{\Delta \rightarrow 0} \frac{\varepsilon(\Delta)}{\Delta}, \\
& \Lambda_{s}=\pi_{s} \lim _{\Delta \rightarrow 0} \frac{c\left(q_{s}(t)+\varepsilon(\Delta)\right)-c\left(q_{s}(t)\right)}{\varepsilon(\Delta)} \frac{\varepsilon(\Delta)}{\Delta}=\pi_{s} c^{\prime}\left(q_{s}\right) \lim _{\Delta \rightarrow 0} \frac{\varepsilon(\Delta)}{\Delta} .
\end{aligned}
$$

Since $\Lambda_{b}$ and $\Lambda_{s}$ are bounded, $\lim _{\Delta \rightarrow 0} \varepsilon(\Delta) / \Delta$ must have a limit. Thus, $\varepsilon(\Delta)$ converges to zero at rate $\Delta$ as $\Delta \rightarrow 0$.

Lemma 4 states that in the limit as $\Delta \rightarrow 0$, it does not matter who makes an offer. This is because the first-mover advantage disappears as the interval between offers becomes negligible. In what follows, I denote $q \equiv q_{b}=q_{s}$.

With this result, let us complete a proof of Proposition 1. In the limit as $\Delta \rightarrow 0$, (3) and (4) are:

$$
\begin{aligned}
& -\pi_{b} U^{\prime}(q) \lim _{\Delta \rightarrow 0} \frac{\varepsilon(\Delta)}{\Delta}=-(M+r) U(q)+M V_{b}-(M+r) V_{s}+U^{\prime}(q) \dot{q}+\dot{V}_{s}, \\
& -\pi_{s} c^{\prime}(q) \lim _{\Delta \rightarrow 0} \frac{\varepsilon(\Delta)}{\Delta}=(1-M+r) c(q)+(1-M) V_{s}-(1-M+r) V_{b}-c^{\prime}(q) \dot{q}+\dot{V}_{b} .
\end{aligned}
$$


An important implication of Lemma 4 is that $\lim _{\Delta \rightarrow 0} \varepsilon(\Delta) / \Delta$ is well defined. Thus, one can eliminate $\lim _{\Delta \rightarrow 0} \varepsilon(\Delta) / \Delta$ from these expressions to obtain

$$
\frac{\pi_{b} U^{\prime}(q)}{\pi_{s} c^{\prime}(q)}=\frac{(M+r) U(q)-M V_{b}+(M+r) V_{s}-U^{\prime}(q) \dot{q}-\dot{V}_{s}}{-(1-M+r) c(q)-(1-M) V_{s}+(1-M+r) V_{b}+c^{\prime}(q) \dot{q}-\dot{V}_{b}} .
$$

Use (1) and (2) to rewrite it as

$$
\frac{\pi_{b} U^{\prime}(q)}{\pi_{s} c^{\prime}(q)}=\frac{(1+r)\left[U(q)+V_{s}-V_{b}\right]-U^{\prime}(q(t)) \dot{q}+\dot{V}_{b}-\dot{V}_{s}}{(1+r)\left[-c(q)+V_{b}-V_{s}\right]+c^{\prime}(q) \dot{q}+\dot{V}_{s}-\dot{V}_{b}} .
$$

\section{B Proof of Proposition 2}

In matrix form, the system of differential equations (8)-(9) is given by

$$
\left[\begin{array}{c}
\dot{q} \\
\dot{V}
\end{array}\right]=\left[\begin{array}{c}
G(q) \\
-(1-M) U(q)-M c(q)+(1+r) V
\end{array}\right]
$$

The Jacobian of the system is

$$
J=\left[\begin{array}{cc}
G^{\prime}(q) & 0 \\
-(1-M) U^{\prime}(q)-M c^{\prime}(q) & 1+r
\end{array}\right] .
$$

The determinant of the Jacobian matrix is $\operatorname{det} J=(1+r) G^{\prime}(q)$, and the trace is $\operatorname{tr} J=$ $G^{\prime}(q)+1+r$. Furthermore, $(\operatorname{tr} J)^{2}-4 \operatorname{det} J=\left[G^{\prime}(q)-1-r\right]^{2}>0$. Therefore, a steady state is a saddle if $G^{\prime}(q)<0$ and is a source if $G^{\prime}(q)>0$. See, for example, Azariadis (1993, p.135).

From (9), it is easy to obtain

$$
\begin{aligned}
G^{\prime}(q) & =\pi_{s} \frac{(M+r) U^{\prime}(q)-M c^{\prime}(q)}{U^{\prime}(q)}-\pi_{b} \frac{(1-M) U^{\prime}(q)-(1-M+r) c^{\prime}(q)}{c^{\prime}(q)} \\
& -\pi_{s} \frac{[(M+r) U(q)-M c(q)] U^{\prime \prime}(q)}{U^{\prime}(q)^{2}}+\pi_{b} \frac{[(1-M) U(q)-(1-M+r) c(q)] c^{\prime \prime}(q)}{c^{\prime}(q)^{2}} .
\end{aligned}
$$

The last two terms are positive because $(1-M+r) c(q) \leq(1-M) U(q)$ and $M c(q) \leq$ $(r+M) U(q)$ are satisfied in any monetary equilibrium. Consider the sum of the first two terms, which is positive if and only if

$$
\begin{aligned}
& \pi_{s}(M+r)-M \pi_{b} \frac{(1-M) U(q)-(1-M+r) c(q)}{(M+r) U(q)-M c(q)} \\
> & (1-M) \pi_{s} \frac{(M+r) U(q)-M c(q)}{(1-M) U(q)-(1-M+r) c(q)}-\pi_{b}(1-M+r),
\end{aligned}
$$

which follows from the fact that the steady state $q$ solves $G(q)=0$, or equivalently:

$$
\frac{U^{\prime}(q)}{c^{\prime}(q)}=\frac{\pi_{s}}{\pi_{b}} \frac{(M+r) U(q)-M c(q)}{(1-M) U(q)-(1-M+r) c(q)} .
$$


Rewrite the condition further to obtain

$$
\frac{[(1-M+r)(M+r)-M(1-M)] \pi_{b} U(q)}{(M+r) U(q)-M c(q)}>\frac{[(M+r)(1-M+r)-(1-M) M] \pi_{s} c(q)}{(1-M) U(q)-(1-M+r) c(q)},
$$

where the denominators of both sides are positive. Notice that $(1-M+r)(M+r)-M(1-$ $M)=(1+r) r>0$. Thus,

$$
\frac{\pi_{b} U(q)}{(M+r) U(q)-M c(q)}>\frac{\pi_{s} c(q)}{(1-M) U(q)-(1-M+r) c(q)},
$$

or

$$
\frac{U(q)}{c(q)}>\frac{\pi_{s}}{\pi_{b}} \frac{(M+r) U(q)-M c(q)}{(1-M) U(q)-(1-M+r) c(q)}=\frac{U^{\prime}(q)}{c^{\prime}(q)} .
$$

Thus, if $U c^{\prime}>U^{\prime} c$, then $G^{\prime}(q)>0$. Notice that $U c^{\prime}>U^{\prime} c \Leftrightarrow \frac{c^{\prime}}{c} q>\frac{U^{\prime}}{U} q$. Since the utility function is assumed to be more concave than the cost function, this condition is satisfied. 


\section{References}

[1] Azariadis, Costas. Intertemporal Macroeconomics, Blackwell, Cambridge, Massachusetts, 1993.

[2] Brock, William A. "A Simple Perfect Foresight Monetary Model." Journal of Monetary Economics 1 (1975) 133-150.

[3] Coles, Melvyn G., and Randall Wright. "A Dynamic Equilibrium Model of Search, Bargaining, and Money." Journal of Economic Theory 78 (1998) 32-54.

[4] Obstfeld, Maurice, and Kenneth Rogoff. "Speculative Hyperinflations in Maximizing Models: Can We Rule Them Out?" Journal of Political Economy 91 (1983) 675-687.

[5] Osborne, Martin J., and Ariel Rubinstein. Bargaining and Markets. Academic Press, San Diego, 1990.

[6] Rubinstein, Ariel. "Perfect Equilibrium in a Bargaining Model." Econometrica 50 (1982) 97-109.

[7] Rubinstein, Ariel, and Asher Wolinsky. "Equilibrium in a Market with Sequential Bargaining." Econometrica 53 (1985) 1133-1150.

[8] Woodford, Michael. Interest $\&$ Prices, Princeton University Press, Princeton, New Jersey, 2003. 